\title{
PIK3C2A is a gene-specific target of microRNA-518a-5p in imatinib mesylate-resistant gastrointestinal stromal tumor
}

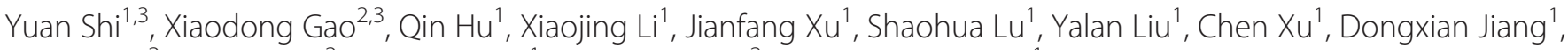 \\ Jiaqian Lin², Anwei Xue ${ }^{2}$, Yunshan Tan', Kuntang Shen² and Yingyong Hou'
}

Imatinib mesylate resistance occurs in some patients with gastrointestinal stromal tumors (GISTs) during the course of treatment. In this study, we investigated the relationship between microRNAs (miRNAs) and imatinib-resistant GISTs, and the effect of miR-518a-5p on PIK3C2A in imatinib-resistant GISTs. A total of 20 matched-pair GIST samples from imatinibresistant patients were included in the study. Each of the paired tumor specimens were from the same patient who had surgical removal of GISTs preimatinib and postimatinib treatment. Seven pairs of tissues were resected for microarray analysis, and the remaining 13 pairs were utilized for miRNAs analysis. Target genes were selected based on bioinformatics from multiple biological databases. Luciferase reporter assays were used to confirm the binding of miR-518a-5p to PIK3C2A 3'UTR. GIST882R-NC, 882R-miR-518a-5p-OE, and 882R-miR-518a-5p-KD cell lines were constructed using lentiviral vectors. miR-518a-5p and PIK3C2A expression in 882R-NC, 882R-OE, and 882R-KD cells was assessed by real-time PCR and western blotting. A cell counting kit was used to detect the influence of miR-518a-5p to cell proliferation. TUNEL staining was applied to detect the influence of miR-518a-5p to cell apoptosis. Microarray analysis showed that miR-518a-5p was downregulated in imatinib-resistant GISTs, and the expression of miR-518a-5p was confirmed with good concordance between real-time PCR and miRNA microarray results. Luciferase reporter assays indicated that miR-518a-5p bound to the PIK3C2A 3'UTR. Compared with 882R-OE, PIK3C2A expression was significantly increased in 882R-KD cells. MiR-518a-5p reduced $882 \mathrm{R}$ proliferation and promoted $882 \mathrm{R}$ apoptosis. In conclusion, PIK3C2A is a gene-specific target of miR-518a-5p in imatinib mesylate-resistant GISTs. Low expression of miR-518a-5p is likely to upregulate PIK3C2A and affect the cellular response to the drug, causing resistance to imatinib in GISTs.

Laboratory Investigation (2016) 96, 652-660; doi:10.1038/labinvest.2015.157; published online 7 March 2016

Gastrointestinal stromal tumors (GISTs) are the most common mesenchymal tumors in the gastrointestinal (GI) tract. To date, approximately $85 \%$ of GISTs are reported to harbor activating mutations in KIT or the homologous receptor tyrosine kinase PDGFRA gene. ${ }^{1}$ Imatinib mesylate (formerly STI571, now referred to as Gleevec in the United States and Glivec in Europe (Novartis)) is a selective inhibitor of certain protein tyrosine kinases: the intracellular ABL kinase, the chimeric BCR-ABL fusion oncoprotein of chronic myeloid leukemia, the transmembrane receptor KIT, and the platelet-derived growth factor receptors. ${ }^{2-5}$ Studies have proved that imatinib is effective for many patients with advanced GISTs. ${ }^{6}$ GISTs are therefore another molecular target therapy model in addition to chronic myoleukemia (CML). However, increasing numbers of studies indicate that primary and secondary resistance may occur during the process of imatinib treatment in GISTs. Correlation between microRNAs (miRNAs) and drug resistance has recently begun to attract the researcher's attention and may be a new mechanism of molecular-targeted drug resistance.

A microRNA is a short RNA molecule found in eukaryotic cells. An miRNA molecule has very few nucleotides (at an average of 22) compared with other RNAs. miRNAs are posttranscriptional regulators that bind to complementary sequences on target mRNA transcripts (mRNAs), usually resulting in translational repression or target degradation and gene silencing. ${ }^{7,8}$ miRNAs have a variety of biological functions, being closely related to growth, development, physiological function, and tumor development. miRNAs

${ }^{1}$ Department of Pathology, Zhongshan Hospital, Fudan University, Shanghai, China and ${ }^{2}$ Department of General Surgery, Zhongshan Hospital, Fudan University, Shanghai, China

Correspondence: Dr Y Hou or Dr K Shen, Department of Pathology, Zhongshan Hospital, Fudan University, Fenglin Road \#180, Shanghai 200032, China.

E-mail: houyingyong@hotmail.com or shen.kuntang@zs-hospital.sh.cn

${ }^{3}$ These authors contributed equally to this work.

Received 1 June 2015; revised 28 September 2015; accepted 12 November 2015 
may have roles similar to oncogenes or tumor-suppressor genes in tumors regulating signaling pathways through the direct effect on target genes, thereby affecting the biological behavior of cells. Research to discover the association between miRNAs and drug resistance is just at the beginning stage. San et al ${ }^{9}$ found 19 miRNAs correlated with imatinib resistance in CML. Our study explored the relationship between selected miRNAs and imatinib-resistant GISTs. miR-518a-5p was found downregulated in imatinib-resistant GISTs by miRNA microarray and real-time PCR. PIK3C2A was confirmed to be a gene-specific target of miR-518a-5p. Results suggested that miR-518a-5p is likely to regulate PIK3C2A and subsequently affect the cellular response to the imatinib in GIST.

\section{MATERIALS AND METHODS}

\section{Cell Lines and Antibodies}

Human 293T (Cat. No. 632180) was purchased from Clontech. Human GIST882 cell line was provided by Professor Christopher Fletcher, Brigham and Women's Hospital, Harvard Medical School, USA. Imatinib-resistant cell line GIST882R was induced from GIST882 cell line by the intermittent concentration gradient doubling method.

\section{Patients and Specimens}

Formalin-fixed paraffin-embedded matched-pair specimens from 20 GIST patients (1998-2013, Zhongshan Hospital, Fudan University, Shanghai, China) were obtained, the clinical and pathological information being listed in Table 1 . All patients in the study had undergone surgery followed by imatinib treatment for a duration of 2-11 years. After the patients developed imatinib resistance, surgical resections were performed again to remove their resistant tumors. Both the preimatinib treatment and imatinib-resistant tissues were included in this study. The gene status of KIT, PDGFRA, and BRAF were detected in all of the above tissues. KIT mutations are listed in Table 1, while neither PDGFRA nor BRAF mutation was found in any of the tissues. Among the 20 patients, 7 pairs of preimatinib treatment and imatinibresistant tissues were utilized for microarray analysis and the remaining 13 pairs were used to test the miRNAs. Clinical information was obtained from the GIST database. This study was approved by the Review Board of the Institute, Zhongshan Hospital, Fudan University.

\section{Microarray Analysis}

Total RNA was harvested using Trizol (Invitrogen) and miRNeasy mini kit (QIAGEN) according to the manufacturer's instructions. After passing RNA quantity measurement using the NanoDrop 1000, the samples were labeled with the miRCURY Hy3/Hy5 Power Labeling Kit and hybridized on the miRCURY LNA Array (v.18.0), containing 3100 capture probes. After rinsing, the slides were scanned using the Axon GenePix 4000B microarray scanner. Scanned images were then imported into the GenePix Pro 6.0 software (Axon) for grid alignment and data extraction. miRNAs replicates were
Table 1 Clinical and pathological information of the 20 GIST patients (Nos.1-7 were utilized for microarray analysis and Nos.8-20 were used to test the miRNAs)

\begin{tabular}{|c|c|c|c|c|c|}
\hline No. & Gender & Age & Primary site & $\begin{array}{l}\text { KIT mutation } \\
\text { of primary } \\
\text { GIST }\end{array}$ & $\begin{array}{l}\text { KIT mutation(s) } \\
\text { of imatinib-resistant } \\
\text { GIST }\end{array}$ \\
\hline 1 & Male & 56 & Gastric & Exon 11 & Exon 11 \\
\hline 2 & Male & 60 & Intestinal & Exon 11 & Exon 11 \\
\hline 3 & Male & 33 & Gastric & Wild & Wild \\
\hline 4 & Male & 66 & Gastric & Exon 11 & Exons 11,13 \\
\hline 5 & Male & 55 & Intestinal & Wild & Exon 17 \\
\hline 6 & Male & 52 & Intestinal & Exon 11 & Exon 11 \\
\hline 7 & Male & 65 & Intestinal & Wild & Exon 11 \\
\hline 8 & Male & 47 & Abdominal cavity & Exon 11 & Exon 11 \\
\hline 9 & Male & 53 & Intestinal & Exon 9 & Exons 9, 17 \\
\hline 10 & Female & 64 & Intestinal & Exon 11 & Exon 11 \\
\hline 11 & Male & 72 & Intestinal & Exon 11 & Exon 11 \\
\hline 12 & Male & 46 & Intestinal & Exon 11 & Exons 11,17 \\
\hline 13 & Male & 33 & Abdominal cavity & Wild & Exons 9, 13 \\
\hline 14 & Female & 62 & Intestinal & Exon 11 & Exons 11, 13 \\
\hline 15 & Male & 62 & Gastric & Exon 11 & Exons 11,13 \\
\hline 16 & Male & 48 & Gastric & Exon 11 & Exons 11,13 \\
\hline 17 & Male & 57 & Intestinal & Exon 11 & Exons 11,13 \\
\hline 18 & Female & 46 & Gastric & Exon 11 & Exons 11, 13 \\
\hline 19 & Female & 48 & Abdominal cavity & Exon 11 & Exon 11 \\
\hline 20 & Female & 40 & Intestinal & Exon 9 & Exon 9 \\
\hline
\end{tabular}

averaged, and all samples with miRNA intensity $\geq 30$ were chosen for calculating normalization factor. Expressed data were normalized using the Median normalization. miRNAs expression with significant differences were identified through Volcano Plot filtering. Hierarchical clustering was performed to show distinguishable miRNA expression profiling among samples.

\section{Quantitative Reverse Transcription-PCR}

miR-518a-5p and PIK3C2A expression was analyzed by using SYBR Premix Ex Taq (TAKARA). In all, $1 \mu \mathrm{g}$ of total RNA was reverse-transcribed by using specific stem-loop reverse transcription (RT) primers and then amplified and detected by using PCR with specific primers and ABI PRISM 7500 Sequence Detection System. U6 was used as an endogenous control for miR-518a-5p and RPL13A was used for PIK3C2A. The miR-518a-5p real-time PCR primers were $5^{\prime}$-CTGC AAAGGGAAGCCCTTTC- ${ }^{\prime}$ and 5'-GTGCAGGGTCCGA GGT-3'. The PIK3C2A real-time PCR primers were $5^{\prime}$-CT TACTCATTGCTTCACCAGTGG-3' and 5'-GCCTCAATC CAGGTCACAGCTA- $3^{\prime}$. The U6 real-time PCR primers were 5'-CTCGCTTCGGCAGCACA-3' and 5'-AACGCTTCACG AATTTGCGT-3'. The RPL13A real-time PCR primers were 
Table 2 Oligonucleotides used for psiCHECK-2 luciferase reporter vector construction

\begin{tabular}{ll}
\hline Name & Sequences $\left(5^{\prime} \rightarrow 3^{\prime}\right)$ \\
\hline PIK3C2AXholF & CCGCTCGAGACTAGTGAATGTTGGAGCTITGGAAGC \\
PIK3C2ANotIR & ATAAGAATGCGGCCGCTTATTCCAGAAATATTTAATACAATCGTAG \\
mutPIK3C2A-1F & ACATTCCTTAAAGTATGTAAGTACGAAACGTAATGCAAATGTACTAGTAATTTCA \\
mutPIK3C2A-1R & TGAAAATTACTAGTACATTTGCATTACGTTCGTACTTACATACTITAAGGAAATGT \\
mutPIK3C2A-2F & CGTACCAAAAAGTAGAGTGGAGCCTGAAACGTCTACTACTATCAATAAATTTAAAT \\
mutPIK3C2A-2R & ATTAAAATTATTGATAGTAGTAGACGTTCAGGCTCCACTCTACTITTGGTACG \\
\hline
\end{tabular}

5'-CTCAAGGTGTTTGACGGCATCC-3' and 5'-TACTTC CAGCCAACCTCGTGAG-3'. Relative quantification analysis was performed using the comparative $\mathrm{C} \mathrm{T}\left(2^{-\Delta \Delta C T}\right)$ method.

\section{miRNAs Target Predictions}

Predicted targets of miR-518a-5p and their sites were analyzed by searching the databases miRDB (http://mirdb. org/miRDB/), microRNA.org (http://www.microrna.org/), TargetScan (http://www.targetscan.org/), miRWalk (http:// www.umm.uniheidelberg.de/apps/zmf/mirwalk/), RNAhybrid (http://bibiserv.techfak.uni-bielefeld.de/rnahybrid/), PICTAR (http://pictar.mdc-berlin.de/), PITA (http://genie.weizmann. ac.il/pubs/mir07/mir07_data.html), and RNA22 (http:// cbcsrv.Watson.ibm.com/rna22.html).

\section{Construction of 882R-NC (Negative Control), 882R-OE (882R-miR-518a-5p-Overexpression), and 882R-KD (882R-miR-518a-5p-Knockdown) Cell Lines}

Lentiviral vectors (pHY-LV-KD5.1, pHY-LV-miR1.2) for overexpression and knockdown of miR-518a-5p (negative control, miR-518a-5p-OE, and miR-518a-5p-KD) were purchased from Hanyin Biotechnology (Shanghai, China). Lentiviral particles were produced via transfection of the lentiviral vectors into $293 \mathrm{~T}$ cells according to the manufacturer's protocol. Packed lentivirus- and negative controlinfected $882 \mathrm{R}$ cells and subsequent stable cell lines were established.

\section{Luciferase Reporter Assay}

DNA fragments of miR-518a-5p-binding sites (wild type and mutant) in the PIK3C2A $3^{\prime}$ UTR (predicted by microrna.org and TargetScan Human 5.1) were synthesized and cloned into the multiple cloning sites (XhoI-NotI) of psiCHECK-2 vector (Promega). All constructions were confirmed by DNA sequencing. The constructions were transfected into $882 \mathrm{R}-$ NC, 882R-OE, and 882R-KD cells. Luciferase activity was measured $48 \mathrm{~h}$ after transfection using the Dual-luciferase reporter assay system (Promega) and Glomax luminometer (Promega). All experimental protocols were performed according to the manufacturer's instructions. DNA fragment sequences are listed in Table 2.

\section{Western Blotting Analysis}

Total cell lysate was prepared in RIPA buffer supplemented with $1 \mathrm{mM}$ PMSF. Protein concentrations were determined by the Bradford assay. Equal amount of proteins were loaded and separated using 10\% SDS-polyacrylamide gel electrophoresis and transferred onto polyvinylidene fluoride membranes. Membranes were blocked in TBS- $0.1 \%$ Tween 20 (TBST) $/ 5 \%(\mathrm{w} / \mathrm{v})$ milk for $2 \mathrm{~h}$ at room temperature. The membranes were then incubated with anti-PIK3C2A or anti$\beta$-actin (Cell Signaling Technology, USA). Proteins on the membranes were detected using the ECL Kit (Thermo Scientific, USA) after washing in TBST, and the blots were quantified with Bio-Rad Gel system (Bio-Rad, USA).

\section{Cell Counting Kit Assay}

$882 \mathrm{R}-\mathrm{NC}, 882 \mathrm{R}-\mathrm{OE}$, and $882 \mathrm{R}-\mathrm{KD}$ cells were cultured in a 96-well plate under regular conditions. At 24, 48, 72, and $96 \mathrm{~h}, 10 \mu \mathrm{l}$ cell counting kit-8 (CCK8, Dojindo Laboratories, Japan) was added and incubated for $2 \mathrm{~h}$ at $37^{\circ} \mathrm{C}$ in a humidified incubator. Optical density value was measured at $450 \mathrm{~nm}$. IC50 were calculated by Logit method.

\section{TUNEL Staining}

882R-NC, 882R-OE, and 882R-KD cells were cultured on coverslips. Cells were harvested and fixed with $4 \%$ paraformaldehyde. Cell apoptosis analysis was performed using a TUNEL Staining Kit (Abcam, USA). Cell nuclei were stained with DAPI. All fluorescent images were examined using a Leica DM3000 microscope and photographed using a DFC 420 camera (Leica, Germany).

\section{Statistical Analysis}

All the statistical analyses were performed with the SPSS v.19.0. Microarray data were analyzed by the paired $t$-test. The data according to normal distribution were analyzed by Student's $t$-test, whereas not according to normal distribution were analyzed by the Wilcoxon signed-rank test, and the expression was represented by M (P25-P75). $P$-value $<0.05$ was considered statistically significant. 


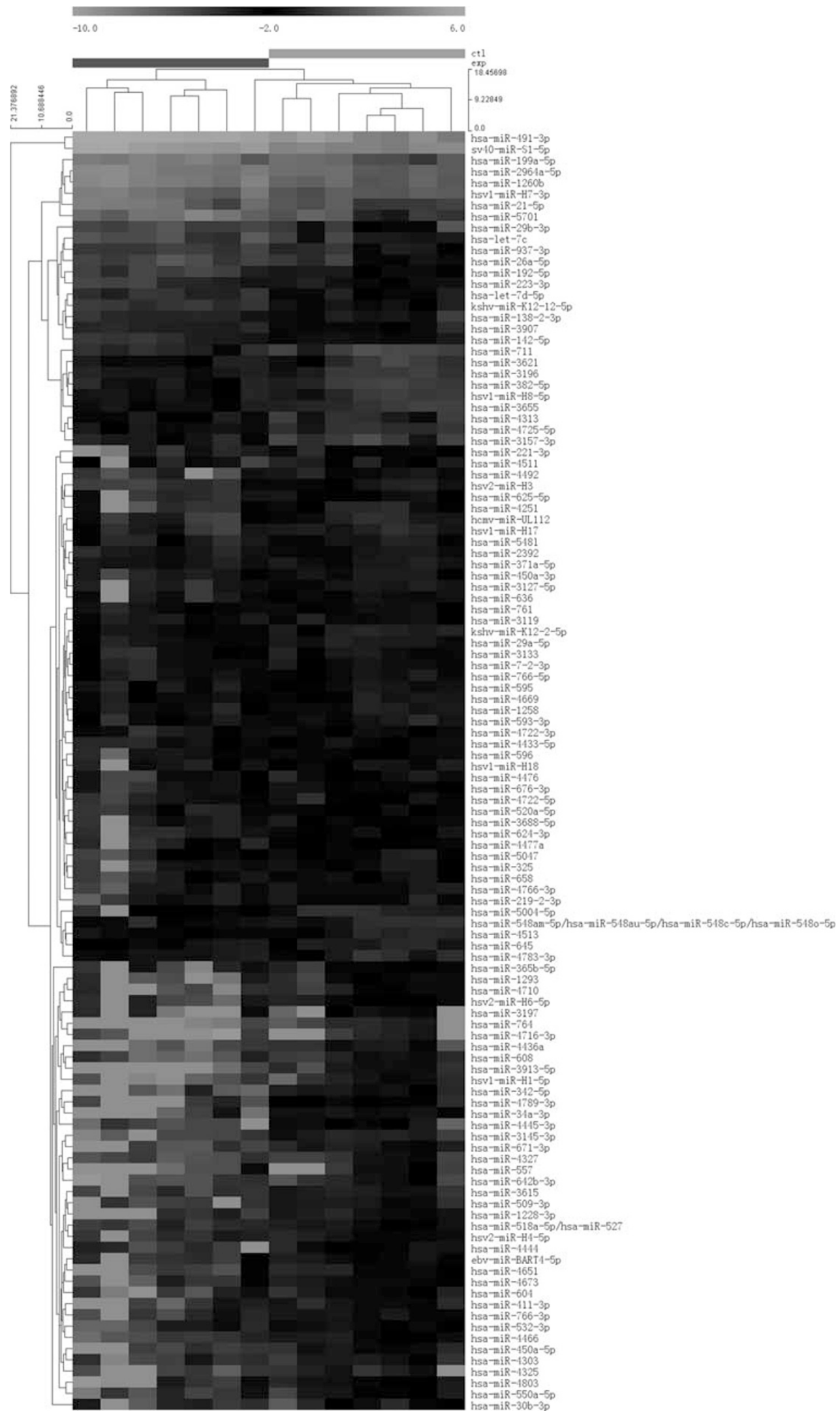

Figure 1 All differentially expressed genes between preimatinib and imatinib-resistant GIST. 


\section{RESULTS}

\section{Microarray Analysis of Preimatinib and Imatinib- resistant GISTs}

Among the 3100 genes analyzed, 114 genes were differentially expressed between preimatinib and imatinib-resistant GIST (Figure 1), and 10 genes were most differentially expressed by at least two folds (fold change $>2$ or $<0.5 ; P<0.05$ ). Compared with the preimatinib GISTs, the expression levels of four miRNAs (hsa-miR-1260b, hsa-miR-2964a-5p, hsamiR-3907, and hsa-miR-491-3p) were upregulated, and the levels of six miRNAs (hsa-miR-518a-5p, hsa-miR-4466, hsamiR-595, hsa-miR-221-3p, hsa-miR-3145-3p, and hsa-miR-3655) were significantly downregulated in imatinib-resistant GISTs (Table 3).

\section{qRT-PCR Analysis of miR-518a-5p in Tissues}

Rubie et $a l^{10}$ in their study found that transfection of colorectal cancer (CRC) cells with miR-518a-5p leads to significant CCR6 downregulation. As CCR6 was found to be upregulated in CRC and involved in CRC pathology, miR-518a-5p might be the tumor suppressor of miRNA. Former microarray analyses showed that miR-518a-5p was downregulated in imatinib-resistant GISTs, so miR-518a-5p was chosen to examine the expression level by qRT-PCR in 13 pairs of preimatinib and imatinib-resistant tissues of GIST. In consistency with microarray data, qRT-PCR results showed that miR-518a-5p expression was reduced in imatinibresistant samples. miR-518a-5p was differentially expressed between the two groups significantly, preimatinib 126306.260 $(19230.355-9061297.280)$ and imatinib-resistant 10.410 $(1.735-2216.285)(P=0.011)$. These data suggested that the downregulation of miR-518a-5p in GISTs might be responsible for the tumor acquiring drug-resistant potential, resulting in imatinib resistance.

\section{The PIK3C2A 3' UTR Contains Binding Sites for miR-518a-5p}

We had previously established the miRNA profile of GISTs by deep sequencing and had discovered that miR-518a-5p was downregulated 2.94-fold in imatinib-resistant GISTs $(P=0.0037)$. These findings had been confirmed by qRTPCR analyses by using a set of 13 corresponding pairs of preimatinib and imatinib-resistant tissues of GISTs. As the mRNA of PIK3C2A (phosphatidylinositol-4-phosphate 3kinase, catalytic subunit type 2 alpha) has been predicted to be a target for miR-518a-5p by a bioinformatics search, we therefore performed a luciferase reporter assay to determine whether miR-518a-5p can directly regulate PIK3C2A expression. In the PIK3C2A 3'UTR, there are two potential binding sites (PBS) for miR-518a-5p, including positions 1999-2005 (PBS1) and 2425-2431(PBS2) (Figure 2). The miR-518a-5pbinding sites were then inserted into the reporter vector and co-transfected with or without miR-518a-5p into GIST882R cells. Compared with $882 \mathrm{R}-\mathrm{NC}$, the luciferase activity was significantly decreased in 882R-OE (fold change: 0.755,

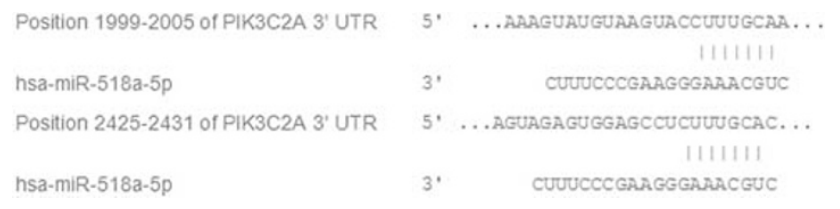

Figure 2 Predicted miRNA target sites on the PIK3C2A 3'UTR. There are two potential binding sites for miR-518a-5p, including positions 19992005 and 2425-2431. Paired sequence alignment is marked by continuous lines.

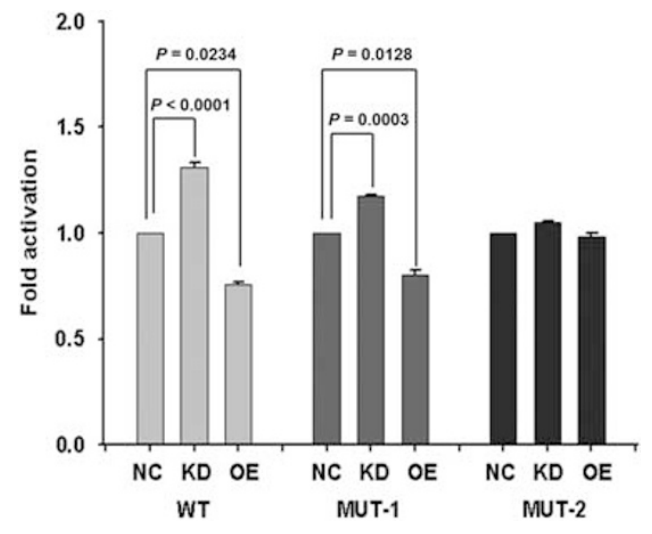

Figure 3 Luciferase activity of WT (wild type, no mutagenesis), MUT-1 (mutagenesis of PBS1), and MUT-2 (mutagenesis of PBS2) in 882R-NC, 882R-OE, and $882 \mathrm{R}-\mathrm{KD}$ cells.

$P=0.0234$ ), while the luciferase activity was significantly increased in 882R-KD (fold change: 1.311, $P<0.0001$ ). Sitedirected mutagenesis (MUT-1 and MUT-2, respectively) was used to remove PBS1 and PBS2. Compared with 882R-NCMUT-1, the luciferase activity was significantly decreased in 882R-OE-MUT-1 (fold change 0.804, $P=0.0128$ ), while the luciferase activity was significantly increased in $882 \mathrm{R}-\mathrm{KD}-$ MUT-1 (fold change: 1.173, $P=0.0003$ ). Compared with 882R-NC-MUT-2, the luciferase activity was no different in 882R-OE-MUT-2 and 882R-KD-MUT-2. This indicated that PBS2 is the target for miR-518a-5p. Taken together, these results suggested that the PIK3C2A $3^{\prime}$ UTR is an independent target for miR-518a-5p (Figure 3).

\section{Relationship Between miR-518a-5p and PIK3C2A Expression}

miR-518a-5p and PIK3C2A expression in $882 \mathrm{~S}$ (imatinibsensitive), 882R, 882R-NC, 882R-OE, and 882R-KD cells were determined using qRT-PCR and western blotting. The result of qRT-PCR showed that, compared with $882 \mathrm{~S}$, miR-518a-5p expression was significantly decreased in $882 \mathrm{R}$ (fold change $0.626, P=0.0007$ ), whereas PIK3C2A was significantly increased (fold change 1.427, $P=0.0003$ ). Compared with 882R-NC, PIK3C2A expression was significantly decreased in $882 \mathrm{R}-\mathrm{OE}$ (fold change $0.416, P<0.0001$ ) and significantly increased in $882 \mathrm{R}-\mathrm{KD}$ cells (fold change $1.234, P<0.0001)$. The result of western blotting showed that, compared with $882 \mathrm{~S}$, PIK3C2A was significantly increased in 
Table 3 Ten most differentially expressed genes between preimatinib and imatinib-resistant GIST

\begin{tabular}{llll}
\hline & P-value & Fold change & $\begin{array}{l}\text { Upregulated or } \\
\text { downregulated }\end{array}$ \\
\hline hsa-miR-1260b & 0.000645 & 2.753673 & Upregulated \\
hsa-miR-2964a-5p & 0.000545 & 2.354872 & Upregulated \\
hsa-miR-3907 & 0.000101 & 3.238698 & Upregulated \\
hsa-miR-491-3p & $5.57 E-05$ & 2.708252 & Upregulated \\
hsa-miR-518a-5p & 0.003665375 & 0.34043 & Downregulated \\
hsa-miR-4466 & 0.000669118 & 0.238874 & Downregulated \\
hsa-miR-595 & 0.001009732 & 0.365527 & Downregulated \\
hsa-miR-221-3p & 0.00163104 & 0.235397 & Downregulated \\
hsa-miR-3145-3p & 0.008019024 & 0.253782 & Downregulated \\
hsa-miR-3655 & 0.000160236 & 0.305696 & Downregulated \\
\hline
\end{tabular}

882R, and compared with 882R-OE, PIK3C2A expression was significantly increased in $882 \mathrm{R}-\mathrm{KD}$ cells (Table 4, Figure 4).

\section{miR-518a-5p Reduced 882R Proliferation}

The effect of miR-518a-5p on the dynamics of $882 R$ growth was determined using a CCK8 assay. Compared with the NC group $(27.41 \pm 0.055)$, the IC50 of $882 \mathrm{R}-\mathrm{OE}(21.27 \pm 0.065)$ and $882 \mathrm{R}-\mathrm{KD}(30.69 \pm 0.039)$ were significantly reduced or promoted, respectively $(P=0.0015$ and $P=0.016$, respectively, Figure 5).

\section{miR-518a-5p Promoted 882R Apoptosis}

To examine the effect of miR-518a-5p on $882 \mathrm{R}$ apoptosis, TUNEL staining was performed. TUNEL staining showed that the proportion of apoptotic cells in $882 \mathrm{R}-\mathrm{OE}$ was increased $(108 \pm 1.65)$ compared with NC group $(70 \pm 1.59)(P<0.0001)$, whereas $882 \mathrm{R}-\mathrm{KD}(47 \pm 1.39)$ cells decreased compared with the NC group $(P<0.0001)$ (Figure 6). These above described results revealed that miR-518a-5p might regulate $882 \mathrm{R}$ proliferation and apoptosis by directly targeting PIK3C2A.

\section{DISCUSSION}

Hirota et $\mathrm{al}^{11}$ reported gain-of-function mutation in KIT gene in GIST in 1998. Heinrich et al ${ }^{12}$ found PDGFRA gene mutation in GIST in 2003. Later, imatinib mesylate was used in GIST treatment. ${ }^{6}$

However, owing to the extended treatment time, drug resistance occurs in some patients, and the median progression-free survival is in the range of 20-24 months. ${ }^{13}$ Although the second-line drug sunitinib and third-line drug sorafenib were introduced into treatment protocols, some patients showed drug resistance after temporary relief, and ultimately treatment failed.

Studies found that miRNAs are closely related to tumorigenesis, invasion, and drug resistance and may have a role as oncogenes or tumor-suppressor genes. Those studies
Table 4 Gene expression of miR-518a-5p and PIK3C2A in 882S, 882R, 882R-NC, 882R-OE, and 882R-KD cells

\begin{tabular}{lcc}
\hline & miR-518a-5p (fold change) & PIK3C2A (fold change) \\
\hline $882 S$ & 1 & 1 \\
$882 R$ & 0.626 & 1.427 \\
$882 R-N C$ & 1 & 1 \\
$882 R-O E$ & 1579 & 0.416 \\
$882 R-K D$ & 0.73 & 1.234
\end{tabular}

provide us new ideas and methods for research in GISTs. miRNAs are non-coding, single-stranded RNAs that function in gene regulation. An miRNA is complementary to a part of one or more mRNAs. Animal miRNAs are usually complementary to a site in the $3^{\prime}$ UTR. The match-ups are imperfect. They regulate gene expression by promoting degradation of the mRNA or repressing its translation. ${ }^{9}$ miRNAs are involved in cell proliferation, apoptosis, differentiation, metabolism, and development of physiological processes, as well as the pathological process of cardiovascular disease, neurological diseases, and cancer metastasis. It is estimated that up to more than one-third of human genes have conserved targets for miRNAs. The roles of miRNAs in human disease, especially in tumors, is an area of active investigation.

In recent years, a large number of studies have shown that miRNAs are involved in tumor development and invasion through regulating the cell cycle, apoptosis, cell migration, angiogenesis, and other tumor-associated gene expression. The relationship between the miRNA and the tumor was first discovered in leukemia. Calin et al ${ }^{14}$ speculated that miRNA may be associated with chronic lymphocytic leukemia. Hereafter, Calin $e a^{15}$ also found that $>50 \%$ of the miRNAs located at fragile sites and genomic regions are involved in cancers. Therefore, miRNAs may have an important role in cancers.

Zhu et $a l^{16}$ first discovered the correlation between miRNAs and drug resistance. They showed that expression of miR-27a and miR-451 were upregulated in multidrugresistant (MDR) cancer cell lines A2780DX5 and KB-V1, as compared with their parental lines A2780 and KB-3-1. They also reported that miRNAs miR-27a and miR-451 are involved in activating the expression of P-glycoprotein, the MDR1 gene product that confers cancer cell resistance to a broad range of chemotherapeutics. About the same time, Xia et $a l^{17}$ found that expression of miR-15b and miR-16 were downregulated in the MDR gastric cancer cell line SGC7901/ VCR compared with its parental SGC7901 cell line. Their findings also suggested that miR-15b and miR-16 could have a role in the development of MDR in gastric cancer cells at least in part by modulation of apoptosis via targeting bcl-2. Inspired by the work of Zhu et all and Xia et al, ${ }^{17}$ San et al ${ }^{9}$ have identified a group of 19 miRNAs that may predict clinical resistance to imatinib in patients with newly diagnosed CML. Among all the possible targets for these 

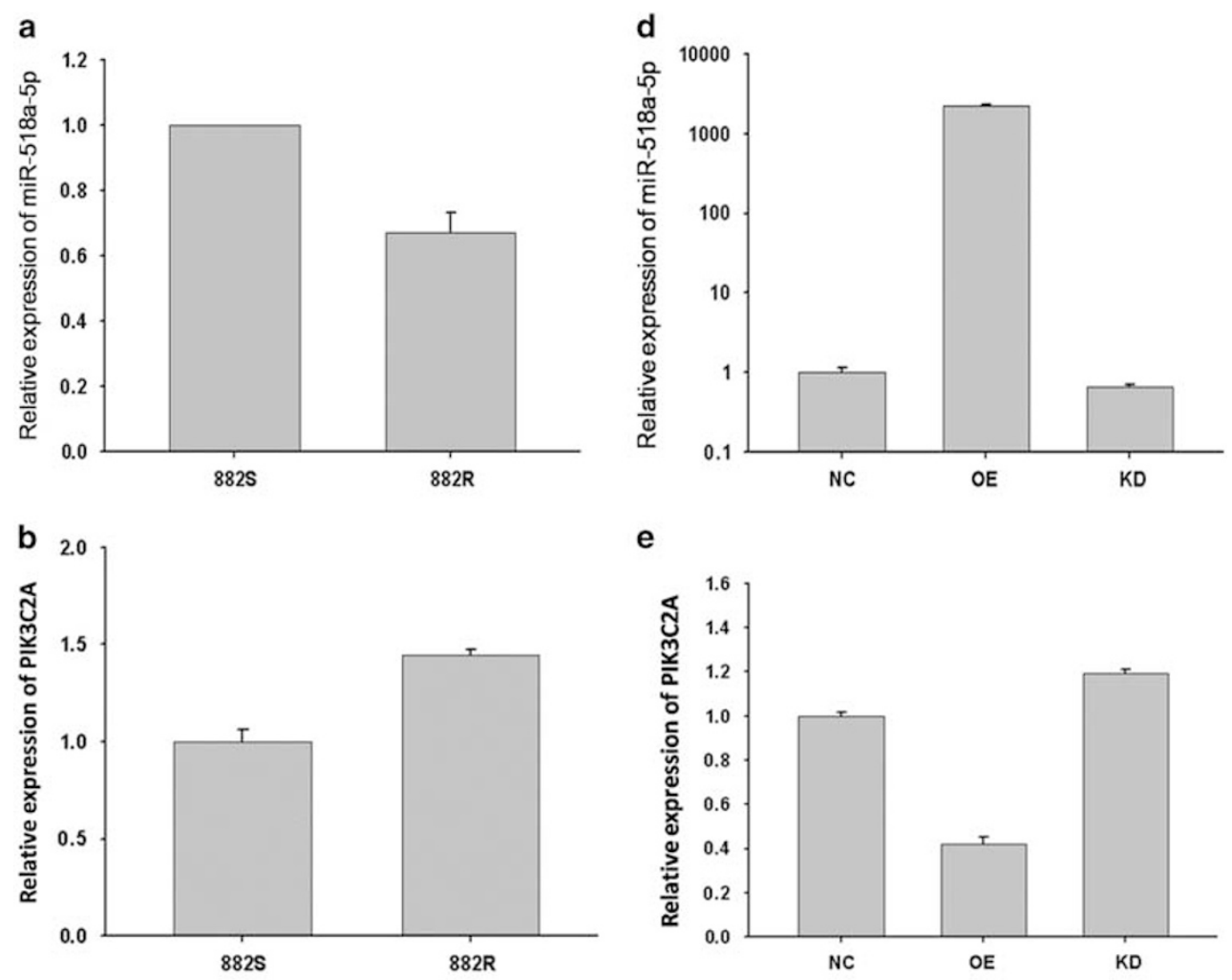

e

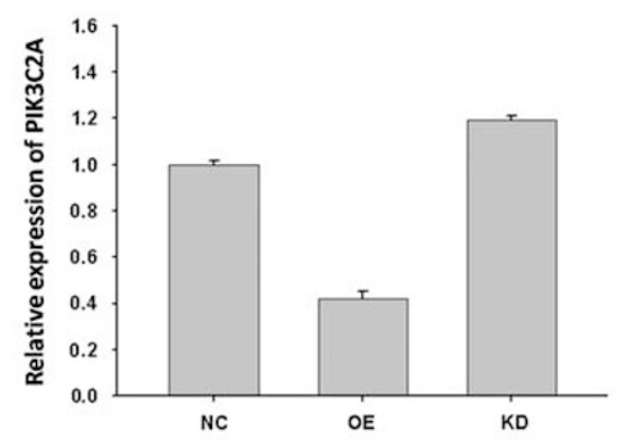

C

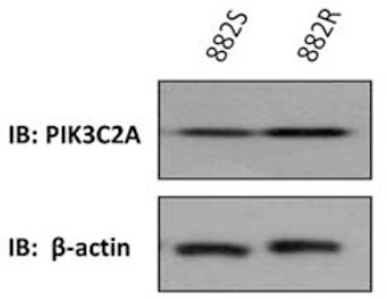

f

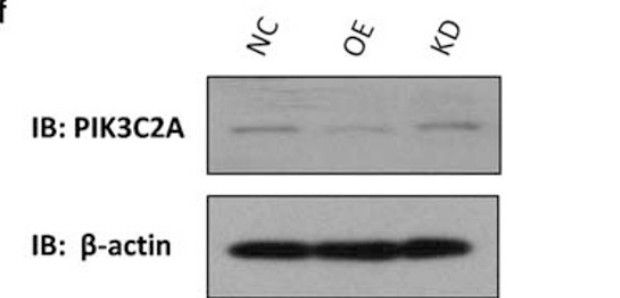

Figure 4 Gene expression of miR-518a-5p and PIK3C2A in 882S, 882R, 882R-NC, 882R-OE, and 882R-KD cells. (a) miR-518a-5p gene expression in 882S and $882 \mathrm{R}$ cells. (b) PIK3C2A gene expression in $882 \mathrm{~S}$ and $882 \mathrm{R}$ cells. (c) PIK3C2A protein expression in $882 \mathrm{~S}$ and $882 \mathrm{R}$ cells. (d) miR-518a-5p gene expression in 882R-NC, 882R-OE, and 882R-KD cells. (e) PIK3C2A gene expression in 882R-NC, 882R-OE, and 882R-KD cells. (f) PIK3C2A protein expression in $882 \mathrm{R}-\mathrm{NC}, 882 \mathrm{R}-\mathrm{OE}$, and $882 \mathrm{R}-\mathrm{KD}$ cells.

miRNAs, they found several membrane transporters that belong to the ATP-binding cassette superfamily of transmembrane transporters have been implicated in resistance to chemotherapy.

Our research team reported previously that miR-320a downregulation is associated with imatinib resistance in GISTs. ${ }^{18}$ However, unmatched-pair samples were used to make microarray analysis, which may cause bias. Therefore, in this study, we used matched-pair samples for microarray and real-time PCR analysis. We found that miR-518a-5p was downregulated in imatinib-resistant GISTs and confirmed that PIK3C2A is the target of miR-518a-5p. Functional interactions between miR-518a-5p and PIK3C2A were investigated.

PIK3C2A is also known as CPK, PI3-K-C2A and PI3-K-C2 (ALPHA). The protein encoded by this gene belongs to the phosphoinositide 3-kinase (PI3K) family. The PI3K pathway

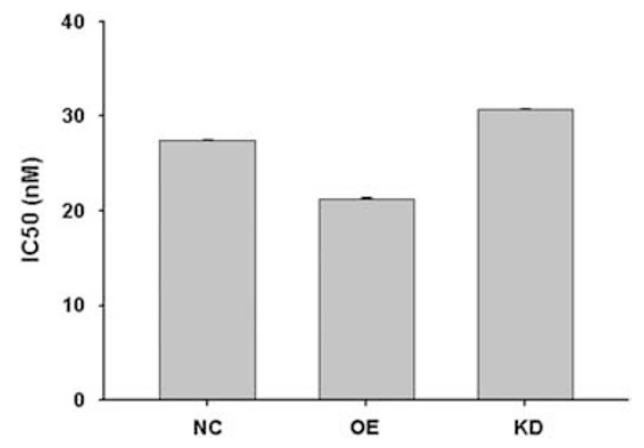

Figure 5 IC50 of $882 \mathrm{R}-\mathrm{NC}, 882 \mathrm{R}-\mathrm{OE}$, and $882 \mathrm{R}-\mathrm{KD}$ cells.

regulates various cellular processes, such as cell growth, proliferation, apoptosis, motility, differentiation, survival, and intracellular trafficking. ${ }^{19}$ PI3 kinases have roles in signaling pathways involved in cell proliferation, oncogenic 

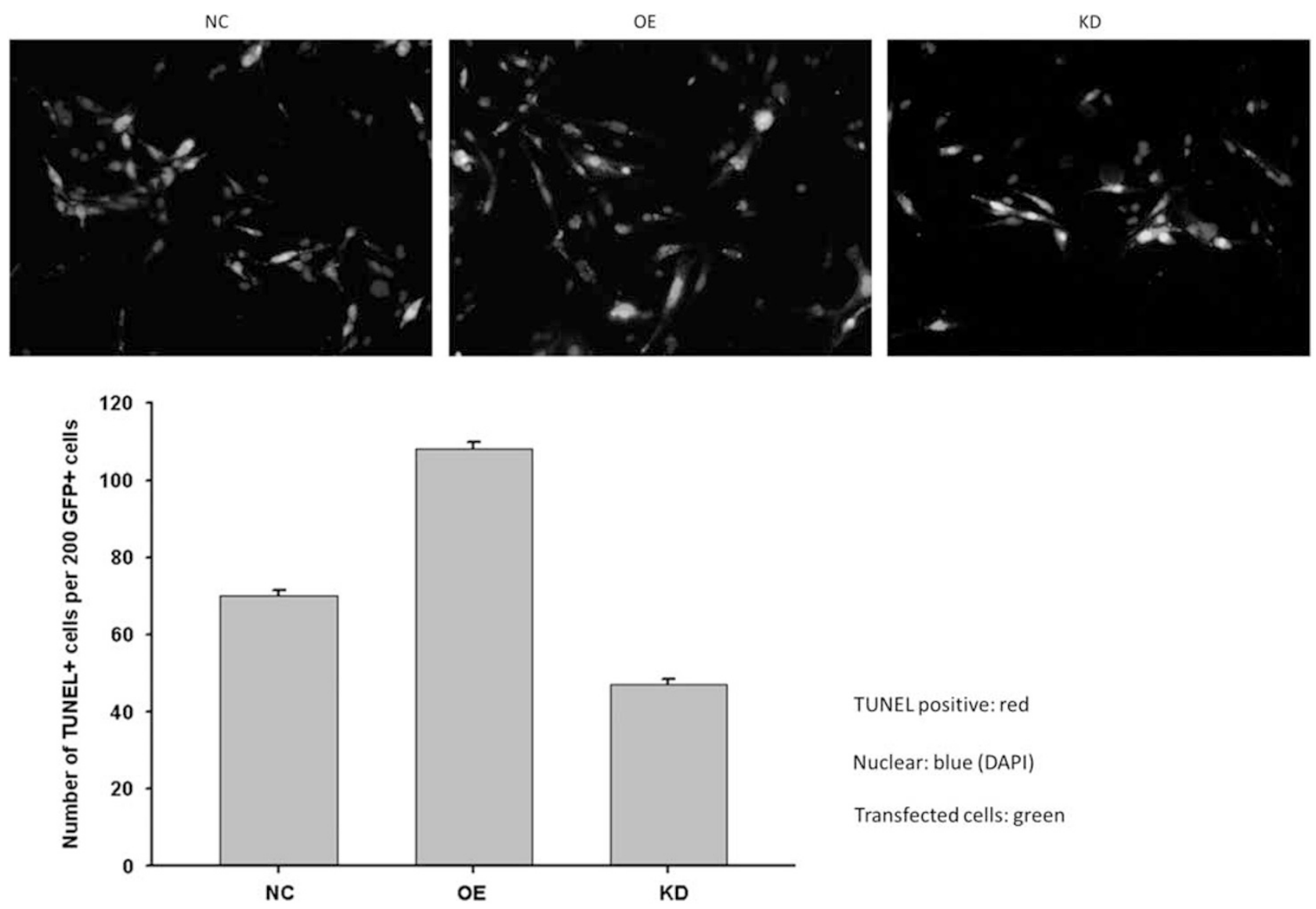

TUNEL positive: red

Nuclear: blue (DAPI)

Transfected cells: green

Figure 6 TUNEL staining of 882R-NC, 882R-OE and 882R-KD cells.

transformation, cell survival, cell migration, and intracellular protein trafficking. This protein contains a lipid kinase catalytic domain as well as a C-terminal C2 domain, a characteristic of class II PI3 kinases. C2 domains act as calcium-dependent phospholipid-binding motifs that mediate translocation of proteins to membranes and may also mediate protein-protein interactions. This protein was shown to be able to be activated by insulin and may be involved in integrin-dependent signaling. The insulin receptor (INSR), via which insulin initiates its signaling, and insulin receptor substrates, which serve as docking proteins binding the PI3K complex, a key regulatory messenger in insulin signaling. ${ }^{20,21}$ $\mathrm{PI} 3 \mathrm{~K}$, via its downstream effector RAC-alpha serine/threonine protein kinase (AKT, otherwise known as protein kinase B or $\mathrm{PKB}$ ), activates several signaling molecules, including mammalian target of rapamycin (MTOR), a promoter of protein synthesis. ${ }^{20-22}$ Rapamycin, an MTOR inhibitor and autophagy inducer, was shown to reduce total and phospho-KIT expression levels and enhance apoptosis in imatinib-resistant GIST cells. ${ }^{23}$ PI3Ks are heterodimeric lipid kinases that are composed of regulatory and catalytic subunits that catalyze the production of several phosphoinositides critical for the signal transduction in these multiple cellular processes. ${ }^{24}$ Mutation, amplification, and rearrangement in the PI3K pathway and its downstream targets have been observed in several cancer sites. ${ }^{25}$ Because of the role of PI3Ks in cell proliferation, much of the research on this pathway concerns its potential as a target for anticancer therapies. ${ }^{26}$ By using our research result for reference, ${ }^{27} \mathrm{Fan} e \mathrm{al}^{28}$ in their study found that the expression of miR-218 is downregulated in an imatinib mesylate-resistant GIST cell line (GIST430), whereas miR-218 overexpression can improve the sensitivity of GIST cells to imatinib mesylate, with PI3K/AKT signaling pathway possibly involved in the mechanism.

There are few studies on miR-518a-5p. To our knowledge, no other investigator has seen miR-518a-5p expression in GIST and we are the first to investigate the relationship between miR-518a-5p and GIST. Our results provide evidence that miR-518a-5p functionally interacts with the $3^{\prime}$ UTR of PIK3C2A. It is further documented that miR-518a-5p reduced $882 \mathrm{R}$ proliferation and promoted $882 \mathrm{R}$ apoptosis, and transfection of GIST cells with miR-518a-5p leads to significant PIK3C2A downregulation at the mRNA and protein level. PIK3C2A has been reported to promote tumor survival, ${ }^{29,30}$ therefore, low expression of miR-518a-5p is likely to upregulate PIK3C2A and affect the cellular response to the drug, causing resistance to imatinib in GIST. Consequently, our findings may aid in the understanding of miRNA gene regulation with 
respect to PIK3C2A and their interaction in GIST cells, emphasizing the importance of further studies of the regulative mechanism underlying the miR-518a-5p/PIK3C2A interaction.

\section{ACKNOWLEDGMENTS}

This research was supported by grants from the National Natural Science Foundation of China (No. 81101809, to Yuan Shi), Shanghai Science and Technology Committee (No. 14411970000, to Yingyong Hou), Shanghai Science and Technology Committee (No. 13411950802, to Yingyong Hou), and Shanghai Municipal Commission of Health and Family Planning, Key developing disciplines (No. 2015ZB0201, to Yingyong Hou). We thank Yingying Lin (Shanghai Jiao Tong University) for technical assistance.

\section{DISCLOSURE/CONFLICT OF INTEREST}

The authors declare no conflict of interest.

1. Liegl-Atzwanger B, Fletcher JA, Fletcher CD. Gastrointestinal stromal tumors. Virchows Arch 2010;456:111-127.

2. Druker BJ, Tamura S, Buchdunger E et al. Effects of a selective inhibitor of the Abl tyrosine kinase on the growth of Bcr-Abl positive cells. Nat Med 1996;2:561-566.

3. Buchdunger $\mathrm{E}$, Cioffi $\mathrm{CL}$, Law $\mathrm{N}$ et al. Abl protein-tyrosine kinase inhibitor STI571 inhibits in vitro signal transduction mediated by c-kit and platelet-derived growth factor receptors. J Pharmacol Exp Ther 2000;295:139-145.

4. Heinrich MC, Griffith DJ, Druker BJ et al. Inhibition of c-kit receptor tyrosine kinase activity by STI 571, a selective tyrosine kinase inhibitor. Blood 2000;96:925-932.

5. Wang WL, Healy ME, Sattler $M$ et al. Growth inhibition and modulation of kinase pathways of small cell lung cancer cell lines by the novel tyrosine kinase inhibitor STI 571. Oncogene 2000;19:3521-3528.

6. Demetri GD, von Mehren M, Blanke CD et al. Efficacy and safety of imatinib mesylate in advanced gastrointestinal stromal tumors. $\mathrm{N}$ Engl J Med 2002;347:472-480.

7. Bartel DP. microRNAs: target recognition and regulatory functions. Cell 2009;136:215-233.

8. Kusenda B, mraz M, Mayer J et al. microRNA biogenesis, functionality and cancer relevance. Biomed Pap Med Fac Univ Palacky Olomouc Czech Repub 2006;150:205-215.

9. San JE, Roman-Gomez J, Jimenez-Velasco A et al. microRNA expression profiling in Imatinib-resistant chronic myeloid leukemia patients without clinically significant ABL1-mutations. Mol Cancer 2009;8:69.

10. Rubie C, Kruse B, Frick VO et al. Chemokine receptor CCR6 expression is regulated by miR-518a-5p in colorectal cancer cells. J Transl Med 2014;12:48.

11. Hirota S, Isozaki K, Moriyama Y et al. Gain-of-function mutations of c-kit in human gastrointestinal stromal tumors. Science 1998;279:577-580.

12. Heinrich $M C$, Corless $C L$, Duensing $A$ et al. PDGFRA activating mutations in gastrointestinal stromal tumors. Science 2003;299:708-710.
13. Rubin $\mathrm{BP}$, Heinrich $\mathrm{MC}$, Corless $\mathrm{CL}$. Gastrointestinal stromal tumour. Lancet 2007;369:1731-1741.

14. Calin $G A$, Dumitru $C D$, Shimizu $M$ et al. Frequent deletions and down-regulation of micro- RNA genes miR15 and miR16 at 13q14 in chronic lymphocytic leukemia. Proc Natl Acad Sci USA 2002;99: 15524-15529.

15. Calin GA, Sevignani $C$, Dumitru CD et al. Human microRNA genes are frequently located at fragile sites and genomic regions involved in cancers. Proc Natl Acad Sci USA 2004;101:2999-3004.

16. Zhu H, Wu H, Liu X et al. Role of MicroRNA miR-27a and miR-451 in the regulation of MDR1/P-glycoprotein expression in human cancer cells. Biochem Pharmacol 2008;76:582-588.

17. Xia L, Zhang D, Du R et al. miR-15b and miR-16 modulate multidrug resistance by targeting BCL2 in human gastric cancer cells. Int J Cancer 2008;123:372-379.

18. Gao X, Shen K, Wang C et al. MiR-320a downregulation is associated with imatinib resistance in gastrointestinal stromal tumors. Acta Biochim Biophys Sin (Shanghai) 2014;46:72-75.

19. Foster FM, Traer CJ, Abraham SM et al. The phosphoinositide (PI) 3-kinase family. J Cell Sci 2003;116:3037-3040.

20. Saltiel AR, Kahn CR. Insulin signalling and the regulation of glucose and lipid metabolism. Nature 2001;414:799-806.

21. Lizcano JM, Alessi DR. The insulin signalling pathway. Curr Biol 2002;12: R236-R238.

22. Alam H, Weck J, Maizels E et al. Role of the phosphatidylinositol-3kinase and extracellular regulated kinase pathways in the induction of hypoxia-inducible factor (HIF)-1 activity and the HIF-1 target vascular endothelial growth factor in ovarian granulosa cells in response to follicle-stimulating hormone. Endocrinology 2009;150:915-928.

23. Hsueh YS, Chang HH, Chiang NJ et al. MTOR inhibition enhances NVPAUY922-induced autophagy-mediated KIT degradation and cytotoxicity in imatinib-resistant gastrointestinal stromal tumors. Oncotarget 2014;5:11723-11736.

24. Fruman DA, Meyers RE, Cantley LC. Phosphoinositide kinases. Annu Rev Biochem 1998;67:481-507.

25. Vivanco I, Sawyers CL. The phosphatidylinositol 3-Kinase AKT pathway in human cancer. Nat Rev Cancer 2002;2:489-501.

26. Koutros S, Schumacher FR, Hayes RB et al. Pooled analysis of phosphatidylinositol 3-kinase pathway variants and risk of prostate cancer. Cancer Res 2010;70:2389-2396.

27. Shi $Y$, Wang $C Z$, Hou $Y Y$ et al. Screening of differentially expressed microRNAs in borderline and malignant gastrointestinal stromal tumors. Zhonghua Bing Li Xue Za Zhi 2013;42:20-25.

28. Fan $\mathrm{R}$, Zhong J, Zheng $\mathrm{S}$ et al. microRNA-218 increase the sensitivity of gastrointestinal stromal tumor to imatinib through PI3K/AKT pathway. Clin Exp Med 2014;15:137-144.

29. Elis W, Triantafellow E, Wolters NM et al. Down-regulation of class II phosphoinositide 3-kinase alpha expression below a critical threshold induces apoptotic cell death. Mol Cancer Res 2008;6:614-623.

30. Ng SK, Neo SY, Yap YW et al. Ablation of phosphoinositide-3-kinase class II alpha suppresses hepatoma cell proliferation. Biochem Biophys Res Commun 2009;387:310-315. 